Audiology

Neurotology
Audiol Neurotol 2016;21:127-131

DOI: $10.1159 / 000444740$
Received: November 18, 2015

Accepted after revision: February 17, 2016

Published online: April 15, 2016

\title{
Sound Source Localization by Normal- Hearing Listeners, Hearing-Impaired Listeners and Cochlear Implant Listeners
}

\author{
Michael F. Dorman ${ }^{a}$ Louise H. Loiselle ${ }^{a}$ Sarah J. Cook ${ }^{a}$ William A. Yost ${ }^{a}$ \\ René H. Gifford ${ }^{\text {b }}$ \\ ${ }^{a}$ Arizona State University, Tempe, Ariz., and ${ }^{b}$ Vanderbilt University, Nashville, Tenn., USA
}

\section{Key Words}

Cochlear implants $\cdot$ Sound source localization

\begin{abstract}
Objective: Our primary aim was to determine whether listeners in the following patient groups achieve localization accuracy within the 95th percentile of accuracy shown by younger or older normal-hearing $(\mathrm{NH})$ listeners: (1) hearing impaired with bilateral hearing aids, (2) bimodal cochlear implant $(\mathrm{Cl})$, (3) bilateral $\mathrm{Cl}$, (4) hearing preservation $\mathrm{Cl}$, (5) single-sided deaf $\mathrm{Cl}$ and (6) combined bilateral $\mathrm{Cl}$ and bilateral hearing preservation. Design: The listeners included 57 young $\mathrm{NH}$ listeners, 12 older NH listeners, 17 listeners fit with hearing aids, 8 bimodal $\mathrm{Cl}$ listeners, 32 bilateral $\mathrm{Cl}$ listeners, 8 hearing preservation $\mathrm{Cl}$ listeners, 13 single-sided deaf $\mathrm{Cl}$ listeners and 3 listeners with bilateral $\mathrm{Cls}$ and bilateral hearing preservation. Sound source localization was assessed in a sound-deadened room with 13 loudspeakers arrayed in a 180-degree arc. Results: The root mean square (rms) error for the $\mathrm{NH}$ listeners was 6 degrees. The 95th percentile was 11 degrees. Nine of 16 listeners with bilateral hearing aids achieved scores within the 95th percentile of normal. Only 1 of $64 \mathrm{Cl}$ patients achieved a score within that range. Bimodal $\mathrm{Cl}$ listeners scored at a level near chance, as did the listeners
\end{abstract}

with a single $\mathrm{Cl}$ or a single $\mathrm{NH}$ ear. Listeners with (1) bilateral $\mathrm{Cls}$, (2) hearing preservation $\mathrm{Cls}$, (3) single-sided deaf $\mathrm{Cls}$ and (4) both bilateral $\mathrm{Cls}$ and bilateral hearing preservation, all showed rms error scores within a similar range (mean scores between 20 and 30 degrees of error). Conclusion: Modern Cls do not restore a normal level of sound source localization for $\mathrm{Cl}$ listeners with access to sound information from two ears.

(C) 2016 S. Karger AG, Basel

\section{Introduction}

A very large number of studies in the literature describe the ability of normal-hearing $(\mathrm{NH})$ listeners to localize sound sources on the horizontal plane [Stevens and Newman, 1936; Middlebrooks and Green, 1991; Blauert, 1997]. Other, more recent, studies describe the ability of patients fit with CIs to localize sound sources on the horizontal plane [van Hoesel and Tyler 2003; Grantham et al. 2007; Seeber and Fastl, 2008]. A major difficulty in comparing the data collected from CI patients with data collected from NH listeners is that the studies have, in general, used different numbers and spacing of loudspeakers, signals with different spectral and temporal characteris-

\section{KARGER}

E-Mail karger@karger.com

www.karger.com/aud
(C) 2016 S. Karger AG, Basel

$1420-3030 / 16 / 0213-0127 \$ 39.50 / 0$
Michael F. Dorman, $\mathrm{PhD}$

Department of Speech and Hearing Science

Arizona State University

Tempe, AZ 85287-0102 (USA)

E-Mail mdorman@asu.edu 
tics, and different measures of root mean square (rms) error. These same problems plague attempts to compare data amongst the many studies with CI patients.

In a first step to remedy this problem, we described the sound source localization ability of a large number of young NH listeners [Yost et al., 2013]. Using the same test environment and stimuli, we tested five groups of CI patients: (1) bimodal listeners, i.e. CI patients with low-frequency hearing in the contralateral ear [Dorman et al., 2015], (2) bilateral CI recipients [Dorman et al., 2014], (3) hearing preservation patients, i.e. patients with a single CI and bilateral low-frequency, acoustic hearing [Loiselle et al., 2015], (4) single-sided deaf (SSD) patients fit with a CI [Dorman et al., 2015; Zeitler et al., 2015] and (5) a patient with bilateral CIs and bilateral low-frequency hearing preservation [Dorman et al., 2013].

In this paper, we compile our previous results with CI patients (increasing the sample size for two of the groups), we increase the sample size for young NH listeners, and we add two new comparison groups: older listeners with ageappropriate hearing and older listeners with sensory hearing loss who are fit with bilateral hearing aids. Our aim was to determine whether any listener in any patient group achieved localization accuracy within the 95th percentile of accuracy shown by younger or older NH listeners.

\section{Methods}

\section{Subjects}

Adult participants ranged in age from 21 to 87 years; 1 child, aged 14 years, also participated. Participants were tested following approval by the IRB at Arizona State University. The work was conducted at the Arizona State University.

The young NH group was comprised of 57 participants (15 male, 42 female) between the ages of 21 and 40 years. Of these, 45 were from the sample described by Yost et al. [2013]. The group of older listeners with age-appropriate hearing was comprised of 12 participants ( 4 male, 8 female) between the ages of 51 and 70 years. These listeners had symmetrical thresholds with no more than a 30 - $\mathrm{dB}$ loss through $2 \mathrm{kHz}$. The mean audiometric thresholds at $0.125,0.25,0.5,1.0,2.0$ and $4.0 \mathrm{kHz}$ were $9,9,7,9,11$ and $23 \mathrm{~dB}$ HL, respectively.

The bilateral hearing-aided group was comprised of 17 participants ( 9 female, 8 male) with symmetrical hearing loss between the ages of 40 and 87 years. Mean right-ear unaided thresholds at 0.25 , $0.5,1.0,2.0$ and $4.0 \mathrm{kHz}$ were $23,31,48,62$ and $72 \mathrm{~dB} \mathrm{HL}$, respectively. The corresponding mean thresholds for the left ear were 24, $33,44,63$ and $74 \mathrm{~dB}$ HL. The participants wore hearing aids of the same make and model on both ears and each had been verified to provide NAL-NL1 target audibility for 60- and 70-dB-SPL speech.

The bimodal CI group was comprised of 8 participants ( 4 male, 4 female) between the ages of 42 and 87 years. The devices used included 2 Advanced Bionics, 4 Cochlear Corporation and 2
MED-EL. The group's mean unaided thresholds in the ear contralateral to the CI at $0.25,0.5,1.0,2.0$ and $4.0 \mathrm{kHz}$ were $50,56,75,96$ and $104 \mathrm{~dB} \mathrm{HL}$, respectively. All hearing aids were verified to ensure NAL-NL1 target audibility for 60 - and $70-\mathrm{dB}-\mathrm{SPL}$ speech. The participants were previously described in Dorman et al. [2015].

The bilateral CI group was comprised of 32 participants (13 male, 19 female) between the ages of 32 and 79 years. The devices used included 7 Advanced Bionics, 9 Cochlear Corporation and 16 MED-EL. Of the 32 listeners, 16 were from the sample in Dorman et al. [2014].

The hearing preservation CI group was comprised of 8 participants ( 4 male, 4 female) between the ages of 35 and 79 years with symmetrical (no more than a $15-\mathrm{dB}$ difference at $250 \mathrm{~Hz}$ ) lowfrequency acoustic hearing in the CI ear and in the contralateral ear. The mean unaided audiometric thresholds for the nonimplanted ear at $0.125,0.25,0.5,1.0,2.0$ and $4.0 \mathrm{kHz}$ were $26,26,46$, 74,105 and $114 \mathrm{~dB}$ HL, respectively. The mean unaided audiometric thresholds for the implanted ear at $0.125,0.25,0.5,1.0,2.0$ and $4.0 \mathrm{kHz}$ were 27, 31, 58, 86, 116 and $124 \mathrm{~dB}$ HL, respectively. The devices used were 3 Cochlear Corporation and 5 MED-EL. All listeners were from the sample in Loiselle et al. [2015].

The SSD group was comprised of 13 participants ( 6 male, $7 \mathrm{fe}$ male) between the ages of 11 and 63 years. The devices used were 1 Advanced Bionics, 1 Cochlear Corporation and 11 MED-EL. Four of the listeners were from the sample in Dorman et al. [2015].

The group with bilateral CIs and bilateral hearing preservation (bi-bi) was comprised of 3 participants ( 2 male, 1 female) between 36 and 53 years of age. The devices used included 1 Cochlear Corporation and $2 \mathrm{MED}-\mathrm{EL}$. The mean right-ear unaided audiometric thresholds at $0.25,0.5,1.0,2.0$ and $4.0 \mathrm{kHz}$ were $25,63,97,108$ and $118 \mathrm{~dB}$ HL, respectively. The corresponding mean thresholds for the left ear were 32, 70, 95, 100 and $120 \mathrm{~dB}$ HL. One of the participants was described in Dorman et al. [2013].

\section{Test Environment and Stimuli}

The environment and methods have been previously described in detail in Yost et al. [2013]. The stimuli were presented in the frontal horizontal plane using a 13-loudspeaker array with 15-degree spacing. The stimuli consisted of $200-\mathrm{ms}$ Gaussian noise bursts filtered between 125 and 6,000 Hz (48 dB/octave).

\section{Test Conditions}

Each stimulus was presented 4 times per loudspeaker at 65 dBA. Stimuli were not presented from either of the end speakers ( 1 and 13 ) along the array.

Prior to testing, the stimuli were presented to ensure audibility and to confirm understanding of the task. During this pretesting period, the patients were allowed to make adjustments to their hearing aid and/or CI volume to ensure comfortable loudness for the 65-dBA stimuli.

Each subject identified the speaker of the sound source by pushing a button on a numbered keypad corresponding to the number of the loudspeaker. The subjects were instructed to return to midline ('look at the red dot on speaker 7') as soon as they pressed the enter button so that they would be positioned in the center when the next stimulus was presented.

Localization accuracy was calculated in terms of rms error using the D statistic of Rakerd and Hartman [1986]. Chance performance, calculated using a Monte Carlo method, was 73.5 degrees $(\mathrm{SD}=3.2)$. 
Fig. 1. Sound source localization accuracy for $\mathrm{NH}$, hearing-impaired and CI listeners. Each symbol indicates the performance of 1 listener. In the single ear condition, the half-filled symbols show the performance of SSD-CI patients when using their $\mathrm{NH}$ ear and the open circles show the performance of the same patients using their CI alone. The dotted line indicates the 95th percentile of scores for the $\mathrm{NH}$ listeners.

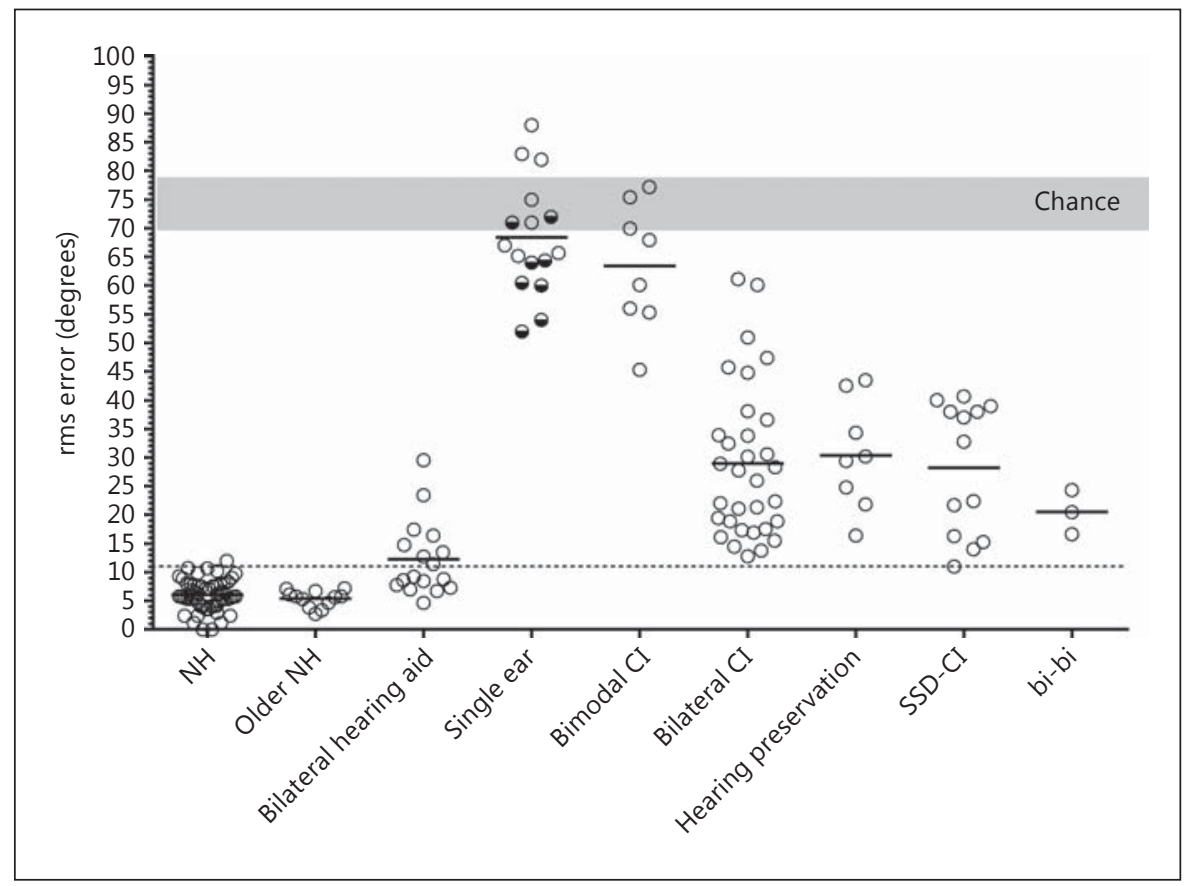

\section{Results}

Localization accuracy, in rms error, is plotted for all patients in all groups in figure 1.

\section{NH Listeners: Younger and Older}

The mean rms error for the younger $\mathrm{NH}$ listeners was 6.0 degrees; the mean rms error for the older listeners was 5.4 degrees. The scores for the older listeners were entirely contained within the range of scores for the younger listeners. The 95th percentile of rms error scores for the younger $\mathrm{NH}$ listeners was 11 degrees and is indicated by a dotted horizontal line in figure 1.

\section{Listeners with Bilateral Hearing Aids}

The mean rms error for this group was 12 degrees. Nine of the 16 listeners achieved scores at, or less than, the 95th percentile for young NH listeners. Unaided audiometric thresholds did not account for the variation in rms error scores.

\section{Listeners with a Single CI or a Single NH Ear}

Eight of 13 SSD-CI patients were tested using their CI alone and with their $\mathrm{NH}$ ear alone. In figure 1 the open circles show performance with a single $\mathrm{CI}$ and the halffilled circles show performance with a single $\mathrm{NH}$ ear. The overall mean rms error score was 68 degrees. Scores with a single CI straddle the mean score for chance performance. Scores for the single NH ear tended to be slightly better but near chance.

\section{Bimodal Listeners}

The distribution of scores for bimodal listeners, with one exception, overlapped completely the distribution of scores for listeners with a single $\mathrm{CI}$ or a single $\mathrm{NH}$ ear. The mean rms error score was 62 degrees.

\section{Bilateral CI Listeners}

The mean rms error score for bilateral CI listeners was 29 degrees. The 'best' listeners had error scores just above the 95th percentile of scores for the young NH group and error scores that overlapped the 'poorer' scores for the listeners with bilateral hearing aids. Only 3 of the $27 \mathrm{pa}$ tients had scores that overlapped the best scores for bimodal listeners and listeners with a single ear or CI.

\section{Hearing Preservation Listeners}

The mean rms error for hearing preservation listeners was 30 degrees. The distribution of localization scores was similar to that for the listeners with bilateral CIs.

\section{SSD-CI Listeners}

The mean rms error for the CI recipients with SSD was 28 degrees. The distribution of scores was similar to that 
found for bilateral listeners (minus the patients at the very end of the distribution) and for hearing preservation listeners. One patient achieved a score at the 95th percentile of $\mathrm{NH}$.

\section{bi-bi Listeners}

The scores for the 3 patients in this group were contained within the range below the mean scores for the bilateral CI, hearing preservation CI and SSD-CI listeners. However, the scores were no better than scores for the best listeners in the bilateral CI, hearing preservation CI and SSD-CI groups.

Based on visual inspection of the data, three groups of scores were created: group 1 was composed of the data from younger and older $\mathrm{NH}$ listeners, group 2 was composed of data from the single-ear and bimodal listeners, and group 3 was composed of data from the bilateral, hearing preservation, SSD and bi-bi listeners. A one-way ANOVA for groups was significant $\left(\mathrm{F}_{2,146}=450.5\right.$, $\mathrm{p}<$ 0.0001). Posttests (Holm-Sidak) showed that each group differed from every other group (mean scores: group $1=$ 5.9 , group $2=66.7$ and group $3=28.5$ ).

\section{Discussion}

Localization accuracy varied significantly among, and within, the listener groups. There was a clear separation of performance for three groups of listeners: (1) the $\mathrm{NH}$ listeners and the hearing-impaired listeners fit with hearing aids, (2) single-ear listeners or bimodal CI listeners and (3) bilateral CI, hearing preservation CI, SSD-CI and bi-bi listeners.

\section{$\mathrm{NH}$ and Hearing-Aided Listeners}

The younger and older NH listeners showed the best sound source localization - approximately 6 degrees of rms error on average. The 95th percentile of scores for this group was 11 degrees of error. The majority of listeners with sloping, mild-to-severe bilateral hearing loss showed rms error scores in the range of normal. However, the distribution of scores was clearly different than that shown by the listeners without threshold elevation as only one of the scores was better than the mean score for the listeners in that group, and many were outside of the 95th percentile of normal.

\section{Single-Ear and Bimodal CI Listeners}

Of the listeners with input to two ears, the listeners in the bimodal CI group showed the poorest sound lo- calization performance - no better than the performance of listeners using a single ear. A common account for this outcome is that bimodal CI patients have access to temporal information from the ear with lowfrequency acoustic hearing as well as access to signal level information from the ear fit with a CI. However, neither timing nor level information is well represented at both ears [Tyler et al., 2002; Potts et al., 2009; Dorman et al., 2015].

\section{Bilateral CI, Hearing Preservation CI, SSD-CI and bi-bi Listeners}

The mean scores for these four groups of listeners were similar (between 20 and 30 degrees of error), but small sample sizes for the hearing preservation listeners and the bi-bi listeners preclude a strong comparison of mean performance. The best-performing CI patients in these groups achieved scores that overlapped the poorest scores for the patients fit with bilateral hearing aids. One bi-bi patient was at the 95 th percentile of normal performance.

\section{Conclusion}

Modern CIs do not restore a normal level of sound source localization for CI listeners with access to sound information from two ears. However, as we have previously speculated [Dorman et al., 2015], and consistent with patient report [Bichey and Miyamoto, 2008], the level of localization obtained for all but the bimodal listeners is probably sufficient to use sound sources in a functionally useful way in the real world.

\section{Acknowledgment}

This work was supported by a research grant to M.F.D. and R.H.G (NIDCD R01 DC-010821).

\section{Disclosure Statement}

The authors declare no conflicts of interest. 


\section{References}

Bichey B, Miyamoto R: Outcomes in bilateral cochlear implantation. Otolaryngol Head Neck Surg 2008;138:655-661.

Blauert J: Spatial Hearing. Cambridge, MIT Press, 1997.

Dorman MF, Loiselle L, Stohl J, Yost WA, Spahr A, Brown C, Cook S: Interaural level differences and sound source localization for bilateral cochlear implant patients. Ear Hear 2014; 35:633-640.

Dorman MF, Spahr A, Loiselle L, Zhang T, Cook S, Fiebig P, Yost W: Localization and speech understanding by a patient with bilateral cochlear implants and bilateral hearing preservation. Ear Hear 2013;34:245-248.

Dorman MF, Zeitler D, Cook S, Loiselle L, Yost WA, Wanna GB, Gifford RH: Interaural level difference cues (ILDs) determine sound source localization by single-sided deaf patients fit with a cochlear implant. Audiol Neurotol 2015;20:183-188.
Grantham W, Ashmead D, Ricketts T, Labadie R, Haynes D: Horizontal-plane localization of noise and speech signals by postlingually deafened adults fitted with bilateral cochlear implants. Ear Hear 2007;28:524-541.

Loiselle LH, Dorman MF, Yost WA, Gifford RH: Sound source localization by hearing preservation patients with and without symmetrical low-frequency acoustic hearing. Audiol Neurootol 2015;20:166-171.

Middlebrooks J, Green D: Sound localization by human listeners. Annu Rev Psychol 1991;42: 135-159.

Potts LG, Skinner MW, Litovsky RA, Strube MJ, Kuk F: Recognition and localization of speech by adult cochlear implant recipients wearing a digital hearing aid in the nonimplanted ear (bimodal hearing). J Am Acad Audiol 2009; 20:353-373.

Rakerd B, Hartmann W: Localization of sound in rooms. III. Onset and duration effects. J Acoust Soc Am 1986;80:1695-1706.

Seeber BU, Fastl H: Localization cues with bilateral cochlear implants. J Acoust Soc Am 2008; 123:1030-1042.
Stevens SS, Newman EB: The localization of actual sources of sound. Am J Psychol 1936;48: 297-306.

Tyler RS, Parkinson A, Wilson BS, Witt S, Preece J, Noble W: Patients utilizing a hearing aid and a cochlear implant: Speech perception and localization. Ear Hear 2002;23:98-105.

van Hoesel RJ, Tyler R: Speech perception, localization, and lateralization with bilateral cochlear implants. J Acoust Soc Am 2003;113: 1617-1630

Yost W, Loiselle L, Dorman M, Brown C, Burns J: Sound source localization of filtered noises by listeners with normal hearing: a statistical analysis. J Acoust Soc Am 2013;133:28762882.

Zeitler DM, Dorman MF, Natale SJ, Loiselle L, Yost WA, Gifford RH: Sound source localization and speech understanding in complex listening environments by single-sided deaf listeners after cochlear implantation Otol Neurotol 2015;36:1467-1471. 